\title{
AN UNMANNED AERIAL VEHICLE FOR MULTI-PURPOSE TASKS IN CONSTRUCTION INDUSTRY
}

\author{
Rok Cajzek \\ Gic gradnje d.o.o. \\ Dr Uroš Klanšek \\ University of Maribor, Faculty of Civil Engineering, Slovenia
}

The aim of this paper is to present an unmanned aerial vehicle (UAV) developed for multipurpose usage in construction industry such as construction site monitoring, examination of constructions, infrared thermography, photogrammetry, transport applications and also for marketing activities. Initially, this article briefly introduces the possibilities of UAV applications in construction industry. Thereupon, the paper presents the structure, the hardware and the software system of a hexacopter, specially developed as the UAV for multipurpose use at construction sites and on locations of existing facilities. Due to the nature of the movement and vibrations created during the flight, it was necessary to develop a stabilizing system for capturing quality photos and videos. Therefore, this paper also exposes an applicable solution for such stabilization system which was installed in the UAV. Furthermore, some actual practices and applications performed employing the developed UAV in the field are shown at the end of the paper. The possible uses of the developed UAV demonstrated that it is suitably operational for multipurpose tasks, particularly on the account of its flexibility, generated thrust and installed software system. Additionally, it is unlike the out of the box systems, which are usually intended for single use.

Key words: Unmanned aerial vehicle, UAV, Multitasking, Construction industry, Image stabilization

\section{INTRODUCTION}

Nowadays, significant attention in design and construction of a wide range of facilities is devoted to finding sustainable solutions aimed at reducing costs in construction phases as well as during the object exploitation. On the other hand, with already built facilities, there is less room for manoeuvre in shaping more sustainable ideas. Therefore in the last few years closer attention was paid to actively engaging in development of different systems for effective monitoring of constructions. Timely monitoring and reviewing construction objects can significantly help to reduce cost of repairs, especially in case of large engineering works like towers, bridges, dams and tall buildings.

Traditional inspection methods are mostly based on visual examination of constructions, which are time consuming, expensive and technically difficult. In most cases, special equipment is

needed to perform investigation of certain facility, like scaffoldings, climbing gear and different cranes due to hard to reach locations. With the development of unmanned aerial vehicles (UAVs) the examination technique has changed and became more time and cost efficient.

During the last few years, the use of UAVs has increased sharply and moved from military operations to commercial use for several reasons. One of them is certainly the availability of new technologies that help us construct flying machines for commercial use. A main role goes to different precision sensors like the inertial motion unit (IMU) including gyroscope and accelerometer, which recognize the velocity, orientation and gravitational forces. All the data from different sensors is gathered in the processor unit, which with the assistance of global positioning system (GPS) is capable of autonomous navigation without the significant need for pilot's manual manoeuvring. Important factors in the rapid growth of smaller UAVs are production costs of all these sensors and the accuracy of the GPS for commercial uses.

Application of commercial UAVs on one side simplifies and improves workflow in construction ex- 
amination. However, there are many concerns and negative reviews due to anxieties about privacy and security invasion during the use. In most cases, specific national administration is providing regulations and rules that are often not fully adapted for the use of UAVs, since they are primarily intended for use of conventional aircrafts [1].

In addition to the technologies used for flying, there are several novelties in the field of image stabilization. Such novelties enable us to use UAVs in construction industry for quality visual inspection of different objects. Despite of many advantages of UAVs, construction industry still represents a more conventionally oriented branch, which rather slowly incorporates new technologies into practice.

Hereinafter, this article briefly introduces the possibilities of UAV applications in construction industry. Thereupon, the paper presents the structure, the hardware and the software system of a hexacopter, specially developed as the UAV for multipurpose use at construction sites as well as on locations of existing facilities. Due to the nature of the movement and vibrations created during the flight, it was necessary to develop a stabilizing system for capturing quality photos and videos. Therefore, this paper also exposes an applicable solution for such stabilization system which was installed in the UAV. Finally, some actual practices and applications performed employing the developed UAV in the field are shown at the end of the paper.

\section{POTENTIAL OF UAVS FOR USE IN CON- STRUCTION INDUSTRY}

In construction industry, we are often facing the need for reviewing various structures at hard to reach locations in order to evaluate their condition. Until recently, examination of different hardly accessible constructions was possible with the use of special protective equipment and various tools for lifting, such as various scaffolds, lifting platforms, mobile cranes or other inspection tools. Some of the disadvantages of traditional inspection approaches were the need for specially trained workers (in many cases professional climbers) and the amount of time required for work completion. Furthermore, a wide variety of systems or tools were necessary to review different construction objects and, in many cases, the risk for occupational injuries increased due to work at height. Moreover, in certain situations, it was required to take the partial or complete closures of objects into account to perform contractually agreed works and, in general, it was necessary to deal with the costs associated with closures.

Beside the conventional inspection methods, a variety of different climbing robot techniques was proposed in the past. For example climbing rope robot with a digital camera attached for building surface inspection [2], climbing robot for rough vertical surfaces [3] and quadrupedal robot for pole climbing [4]. Common to all previously mentioned climbing robots is a complex mechanical design to enable movements and limited usability due to diverse shapes of constructions that should be examined. In addition, they usually require considerable set up times and, in many cases, climb slowly, so the inspection job may require quite some time.

On the other hand, different UAV systems were proposed in literature for the aim of inspections, e.g. for bridge inspection [5], for inspection of pole like structures [6], for surveillance and visual inspection [7], for outdoor inspection of building façades [8], for power plant inspections [9] and for monitoring structural damages [10]. Different approaches demonstrate diverse options of using UAVs for commercial purpose of inspection and use in various industries including for research purposes [11]. In spite of different technologies used in above-mentioned UAVs, the basic operational principles remain similar.

As has already been mentioned, the use of UAVs spread from military fields where they were used in various operations during the 1970s and 1980s. Over the years, a range of various types of UAVs has increased significantly and the expansion still rapidly continues. Makers offer a diversity of systems and currently there is no official classification of UAVs for commercial purposes. Regarding the form they can be divided into three major groups; fixed wing aircrafts, unmanned helicopters and multicopters [12]. Table 1 shows a comparison between different types and their main characteristics.

Fixed wing aircrafts are suitable for greater distances due to better energy efficiency and higher speeds. They are applicable for inspecting long, low objects, such as pipelines or roads. Some of the major disadvantages are the space needed for take-off and landing procedure, which is grater in comparison with other types of UAVs and inferior handling capabilities. 
Table 1: Comparison of UAV types and main characteristics

\begin{tabular}{|c|c|c|c|c|c|}
\hline UAV type* & Payload & Range & Handling & Setup time & Costs \\
\hline $\begin{array}{c}\text { Fixed wing } \\
\text { aircraft }\end{array}$ & Good & Good & Poor & Average & $\begin{array}{c}\text { Very } \\
\text { good }\end{array}$ \\
\hline $\begin{array}{c}\text { Unmanned } \\
\text { helicopter }\end{array}$ & $\begin{array}{c}\text { Very } \\
\text { good }\end{array}$ & $\begin{array}{c}\text { Very } \\
\text { good }\end{array}$ & $\begin{array}{c}\text { Very } \\
\text { good }\end{array}$ & Good & Average \\
\hline Multicopter & Average & Average & $\begin{array}{c}\text { Very } \\
\text { good }\end{array}$ & $\begin{array}{c}\text { Very } \\
\text { good }\end{array}$ & Good \\
\hline
\end{tabular}

* There are also other UAV categories (airships, tilt-wing aircrafts, etc.). Types shown above are most common.

The second type of UAVs represents unmanned helicopters that are inspired by military drones and have sophisticated systems for different tasks. The main benefits are the possibility of vertical take-off and landing, extended flight range and the possibility of carrying heavier loads. Such UAVs are well suited for different payload options and multipurpose use; for example, for carrying multiple devices and sensors at the same time on long distances.

On the other hand, they are quite expensive and have higher maintenance costs.

The last type of UAVs used for commercial use represents multicopters. They are possibly the most known in industry due to many strengths. Some of the advantages are low purchase price, ease of manoeuvring, possibility of vertical take-off and landing, low maintenance costs and suitability for various applications. The main deficiencies are low wind resistance, limited payloads and average range due to higher energy consumption.

Due to the nature of works in construction industry, it is necessary to use a UAV with the vertical take-off and landing abilities. In this way, the decision between unmanned helicopter and multicopter was made in favour of the latter because it was more affordable and easier to set up at location. Furthermore, very good handling capabilities, sufficient range and large accessibility of components on the market have further contributed to the decision of using a multicopter.

The UAV was designed and assembled from different components found on the market with the guideline of maximizing possible uses in the field. Despite numerous options for making commercially useable multicopters, the basic components and operating principles are in most cases quite similar among the most available versions. Over the last years, there are numerous options of purchasing UAVs available that are ready to fly (RTF) directly from the box. In many cases, they become very useful and the balance between performance and price is good.

However, in case of professional use, they are not most suitable on account of several limitations. One of the main disadvantages of RTF models is the fact, that they are usually intended only for single purpose use and therefore improvement possibilities are limited. Otherwise, in the near future, we can expect UAVs built on platforms, which will enable an extended multipurpose use.

\section{A UAV FOR MULTIPURPOSE TASKS}

\section{Structure and hardware}

The structure of developed UAV is based on a hexacopter principle, more precisely on hexa-rotor $V$ system. One of the main reasons for selecting a system with six instead of four engines are better reliability and lifting capacity in comparison with quadcopters. Six engines and rotors enable flying thus potential failure on one motor and provide safe landings. The latter is a key advantage in the use of UAVs over populated areas or over construction sites and buildings since it reduces the percentage of collision chances. Using more engines, for example, eight or more does not contribute to a far greater reliability, but in most cases represents a more costly version. Acceptable operating and maintenance costs and possibility of vertical take-off and landing procedure are further benefits of the UAV system that are based on multicopter principles (for example quadcopters, hexacopters and octocopters).

On the other hand, main disadvantages are relatively low wind resistance and limited flight time that is, in this particular case, approximately 25 minutes on average for loaded UAV. Furthermore, in some cases, operating range that is normally up to few hundred meters may also rep- 
resent a limitation. However, that is not of crucial importance, because national regulations often allow only line-of-sight (LOS) of UAVs [13].

The multicopter (see Fig. 1) consists of a carbon fiber frame and landing gears to preserve weight, which enables longer flight times. Screws and lifting mechanism for landing gears are made of aluminium, also to reduce weight.

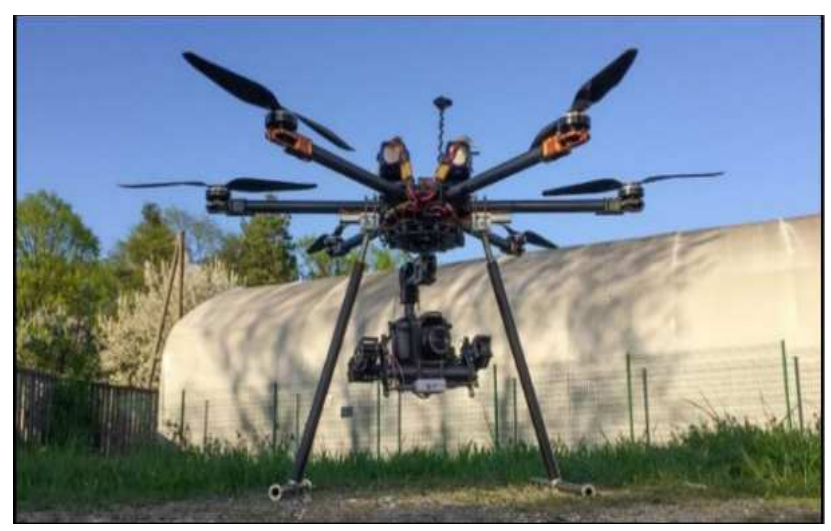

Figure 1: Multicopter for multipurpose use

The powertrain contains six efficient brushless electrical motors, two lithium-polymer six-cell batteries with a total capacity of $8000 \mathrm{mAh}$ $(22.8 \mathrm{~V})$ each and weight of approximately 2 $\mathrm{kg}$ combined. In order to ensure greater safety during the flight and to extend the operating time, two batteries are used during the flight. Maximal absolute thrust of the UAV is $18.4 \mathrm{~kg}$ and it is produced with six $45.72 \mathrm{~cm}$ long carbon fiber propellers, with the total size of 1425 $\mathrm{mm}$ in diameter. Aforesaid average flight time may vary depending on the flying style, wind and load. Total weight of the UAV prepared for take-off is roughly $8 \mathrm{~kg}$ including a camera stabilization system (3-axis gimbal), two batteries and a camera with 14-42 mm lens or similar.

Another important part of the UAV is the flight control unit (FCU), which in our case consists of four different components; a main controller (MC), a power management unit (PMU), a GPS antenna and a LED light. Main function of FCU is to process incoming signals from the remote control $(R C)$ via $R C$ receiver and then communicate with motor controllers (also known as electronic speed controller) as well as with brushless electrical motors, see Figure 2.

For easier flying, there is also the inertial measurement unit (IMU) which detects alignment, barometrical altitude and accelerations of the UAV. In combination with a GPS antenna and a compass, it can fly in three different modes. First mode works with GPS, where the UAV is positioned via satellites and is able to maintain altitude and position in the air. This function is useful in most scenarios while flying is relatively easy and safe due to enabled hold position in the air, so possible winds have smaller effect on the movement of the multicopter. In case of lost GPS signal, altitude mode is enabled after few seconds, to prevent possible failures. Second option of flight type is altitude mode, where UAV is controlled by an altitude meter, by monitoring barometric pressure and this way maintains desired height. The third mode is manual, where the pilot needs to control the UAV completely by himself and without interruption of safety systems.

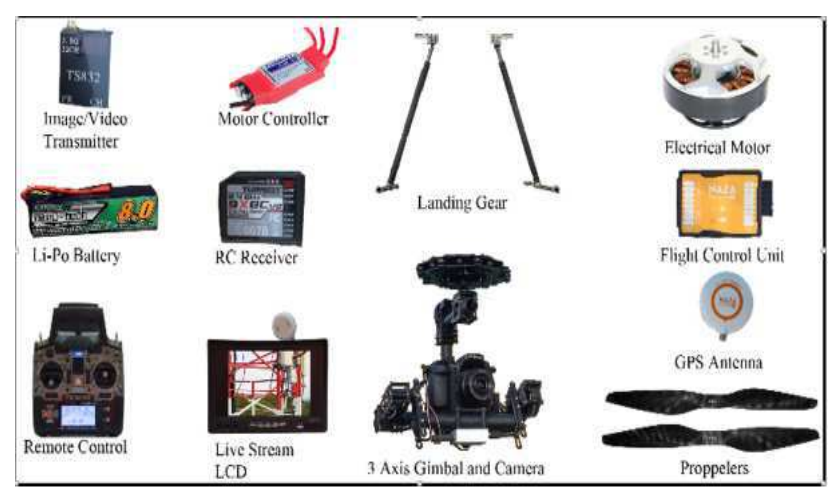

Figure 2: Major components of the developed multicopter (components are not in scale)

Other major components are a video transmitter for sending live picture from multicopter to the pilot's and co-pilot's LCD screen, a three axis camera stabilization system (also known as gimbal) that enables smooth movements and prevents image blur during the flight and electric retractable landing gear for safe landings. Due to powerful and efficient motors on gimbal, it is possible to attach different cameras, including infrared (IR) thermo-camera.

The multicopter can fly with a maximum speed over $20 \mathrm{~m} / \mathrm{s}$ to maximum distance or flight altitude up to two kilometres from the pilot if the area is open (without physical objects like trees, buildings and other barriers). Average traveling speed is around $5 \mathrm{~m} / \mathrm{s}$ and maximum flight altitude usually does not exceed 100 meters.

\section{Camera stabilizing system and software}

Camera stabilizing systems are well known in many different industries and during the last de- 
cades, they have become an irreplaceable tool for aerial image and video capturing. With the invention of camera stabilizer known as Steadicam in the seventies, film industry has changed a lot. With the development of powerful sensors and brushless motors, the stabilizing system significantly improved, however, the operating principle is still the same, to stabilize the image and prevent unwanted vibrations, shake or blur effect. In most cases, gimbal stabilization with brushless motors works for low $(<0.1 \mathrm{~Hz})$ and medium frequencies $(>0.35 \mathrm{~Hz})$ and for small $\left(\Delta<10^{\circ}\right)$ or medium amplitudes $\left(10^{\circ}-25^{\circ}\right)$. For stabilizing higher frequencies, mechanical vibration damping needs to be used. Other possible choices are optical and digital image stabilization systems [14].

Operation of the UAV system is often subject to several sources of vibrations (see Fig. 3); for instance, the FCU induced by the stabilizing task of the UAV at a constant position, vibrations generated by engine and propellers, vibrations caused by nearly constant winds (headwind), wind gusts and also flight manoeuvres performed by the pilot. In general, vibration types can be classified into five segments from the viewpoint of different frequencies and amplitudes.

Lower frequency vibrations with small amplitudes can be produced by stabilizing tasks, for example hovering of the UAV in the air, medium amplitudes by aerodynamic sources, like headwinds and large amplitudes by pilot's manoeuvres of the UAV executed via remote control. Medium frequency vibrations with small amplitudes can be generated by stabilizing tasks or by operating engine and propellers, medium amplitudes by aerodynamic sources and large amplitudes either by flight manoeuvres or by unexpected strong sources, like wind gusts. Higher frequency vibrations with small amplitudes may come from engine and propellers, medium amplitudes partly by aerodynamic sources and large amplitudes by unexpected strong sources.

The 3-axis gimbal applied in the UAV consists of lightweight frame, three brushless electrical motors for controlling pitch, yaw and roll as well as gimbal controller with an IMU for alignments detection, see Fig. 4. For the proper functioning of the gimbal, it is very important that the entire system is balanced in order to reduce the load of individual brushless motor. Stabilizing system operates with a proportional-integral-derivative controller (PID controller), which works as a control loop feedback mechanism. Integral, proportional and derivate feedback is based on the present $(P)$, on the accumulation of past $(I)$ and future prediction (D) control error [15].

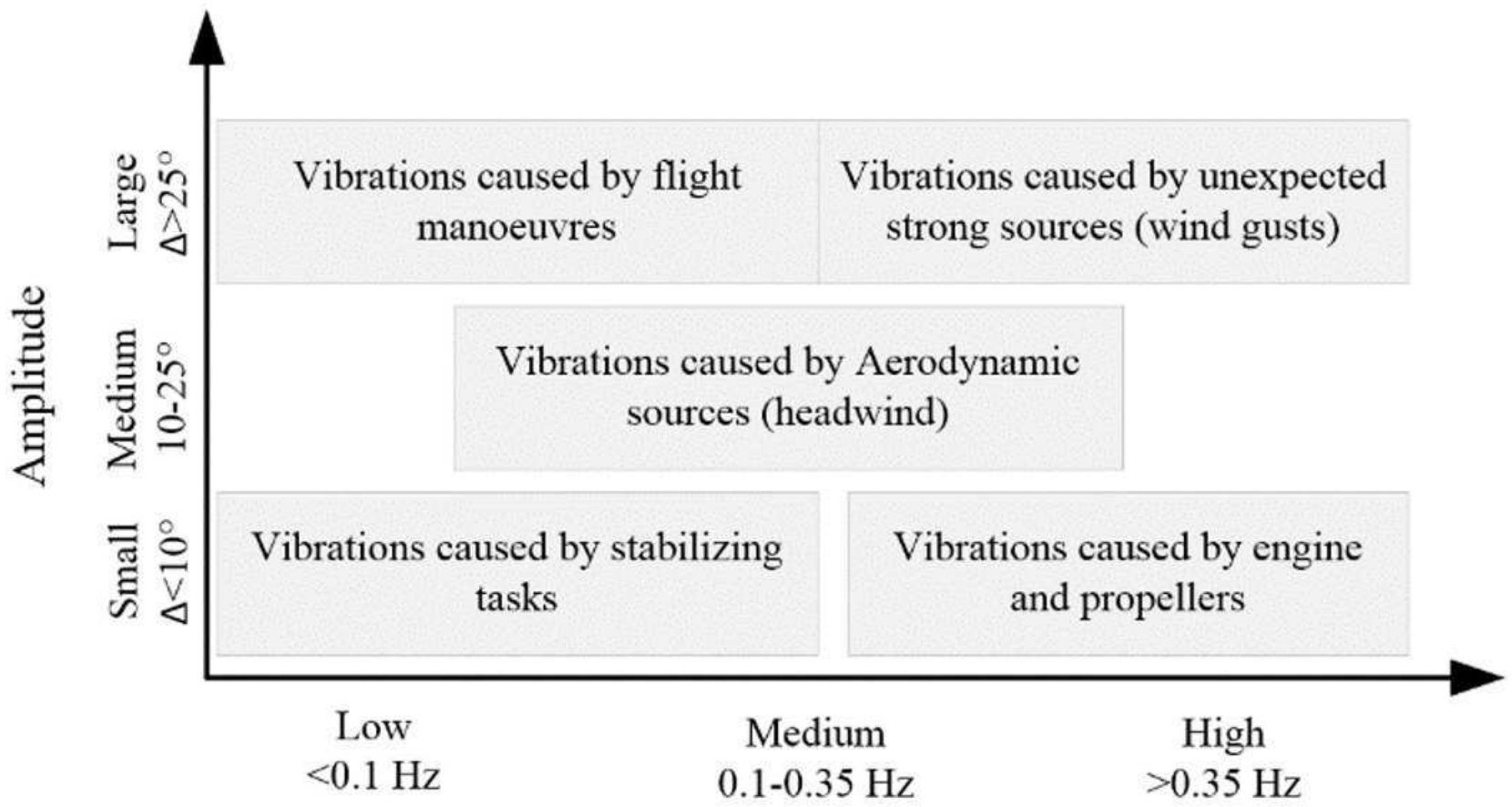

Figure 3: Source of vibrations [14] 


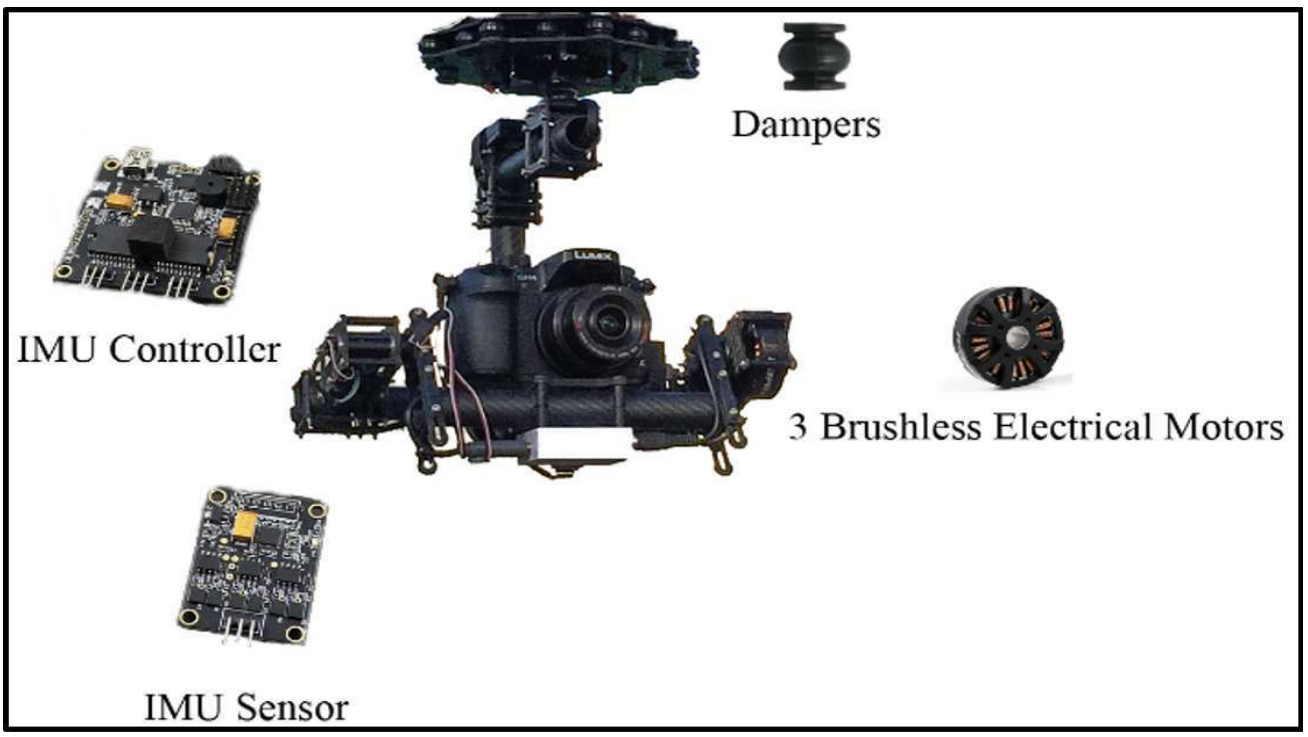

Figure 4: 3-axis gimbal

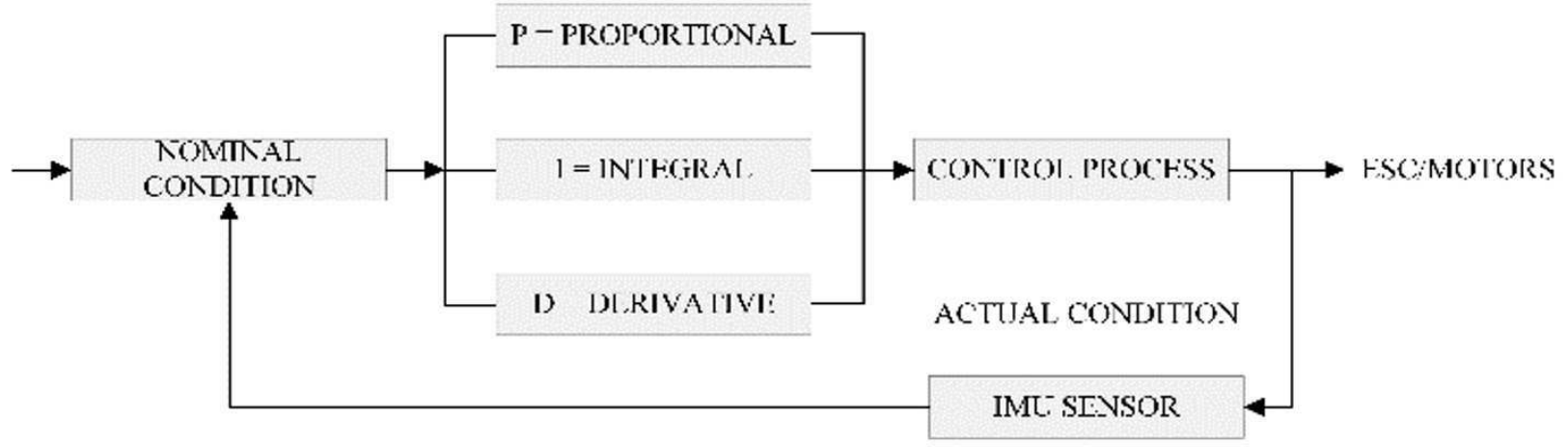

Figure 5: PID controller scheme

Basic principle of the PID controller (see Fig. 5) on gimbal is checking if the actual condition is the same as nominal condition, than there is no need for gimbal motors to start working. In all other cases, if there is a deviation/error in any of three axes (pitch, yaw and roll) from nominal condition, the controller gives a signal (power) to the motors and corrects it. The error is than multiplied by $\mathrm{P}$, which determines the output signal in Volt. The job of the IMU is to send the error and to minimize it; however, it can never perform a complete correction due to correlation between the smaller error and the smaller output power.

In the end, there is not enough power to correct the last few degrees of deviation. With the value I a special interval is set and in this case, the signal is saved for some predefined time. All these savings are summed up and added to the signal (power) and calculated by the $P$ value, so the power never becomes too weak to complete the correction. In many cases, there is a problem with over correction due to too much power created from $P$ and I values, which leads to oscillation. For perfectly smooth picture in the air, there is a need to damp the $P$ and I reaction and that is a job for $D$ part from PID controller.

For a proper functioning of the stabilization system, it is of crucial importance that the PID controller values are set correctly. In our multicopter, we were using an IMU unit with a sensor and an accompanying software [16]. Firstly, it is necessary to set up the correct orientation of the sensor for all six positions. After that, it is required to configure correct values for power and correct number of magnetic poles. Power output values for each motor should be set between 0 and 255, where value 255 represents a full battery voltage sent to a motor. The number of poles is defined by the motor type itself, for regular brushless motors it equals to the number of magnets. After motor configuration, a fine-tuning is needed from $P$ then I and lastly $D$ values for all three axes (pitch, yaw and roll). Finding the right balance for PID controller values takes time and numerous tests of 
stabilization systems equipped with camera and lens need to be performed. The values used in our case are presented in Table 2.

After completing the settings of the stabilization system, we arrange the Low-Pass Filter (LPF) values for enabling smoother reaction between the stick movement and gimbal. By increasing the LPF value, a smoother reaction to the stick can be achieved due to cuts of small RC errors and jitter. To present the functioning of the stabilization system, we installed two IMU gimbal controllers for alignments detection, one on the bottom side of the platform for electronics, and other on the gimbal bellow the camera mount. With the real time data from SimpleBGC software, we were able to monitor values from both locations in order to show the figures of angle deviation in all three axes during the test flight in two different frequency modes.

Operation of the previously described system builtin the UAV was tested with (i) no stabilization, (ii) stabilization with 3-axis gimbal and (iii) stabilization with 3-axis gimbal including the mechanical vibration damping system. The values presented in Fig. 6 show angular deviation for pitch, roll and yaw axis with no stabilization in light grey, with the IMU controller stabilization in dark grey and with both IMU and mechanical rubber dampers in black colour. The left part of Fig. 6 shows low frequency mode, that present slow acceleration to the desired deviation angle and the right part shows medium frequencies, with faster accelerations.

Differences in angular deviation between low and medium frequencies without stabilization are visible in light grey colour (pitch $32^{\circ}$ vs. $31^{\circ}$, roll $29^{\circ}$ vs. $28^{\circ}$ and yaw $90^{\circ}$ vs. $95^{\circ}$ ) on account of difficult repeatability of movements of the UAV for both tests.

Irrespective of the latter, comparable results of angular deviation between all three axes (pitch, yaw and roll) were obtained. Better results (lower angular deviation) at lower frequencies were expected, while the IMU stabilization worked better for slower movements (dark grey colour in Figure $6)$.

For additional damping, rubber dampers were used, which have also contributed to lower angular deviations with their passively damping capabilities (black colour in Figure 6). Effects of stabilization can be seen in all three directions in both cases (low and medium frequencies). At lower frequencies, a $94.49 \%$ reduction of deviation with IMU was achieved in average in all three axes and $97.42 \%$ with both IMU plus dampers. At medium frequencies, a $92.81 \%$ reduction of deviation was gained with IMU and $94.89 \%$ with both IMU plus dampers in average.

Table 2: Main values from SimpleBGC

\begin{tabular}{|c|c|c|c|c|c|}
\hline Axes & P & I & D & Power & No. of poles \\
\hline Pitch axis & 15 & 0,03 & 30 & 160 & 22 \\
\hline Yaw axis & 4 & 0,02 & 8 & 120 & 22 \\
\hline Roll axis & 10 & 0,02 & 19 & 170 & 14 \\
\hline
\end{tabular}

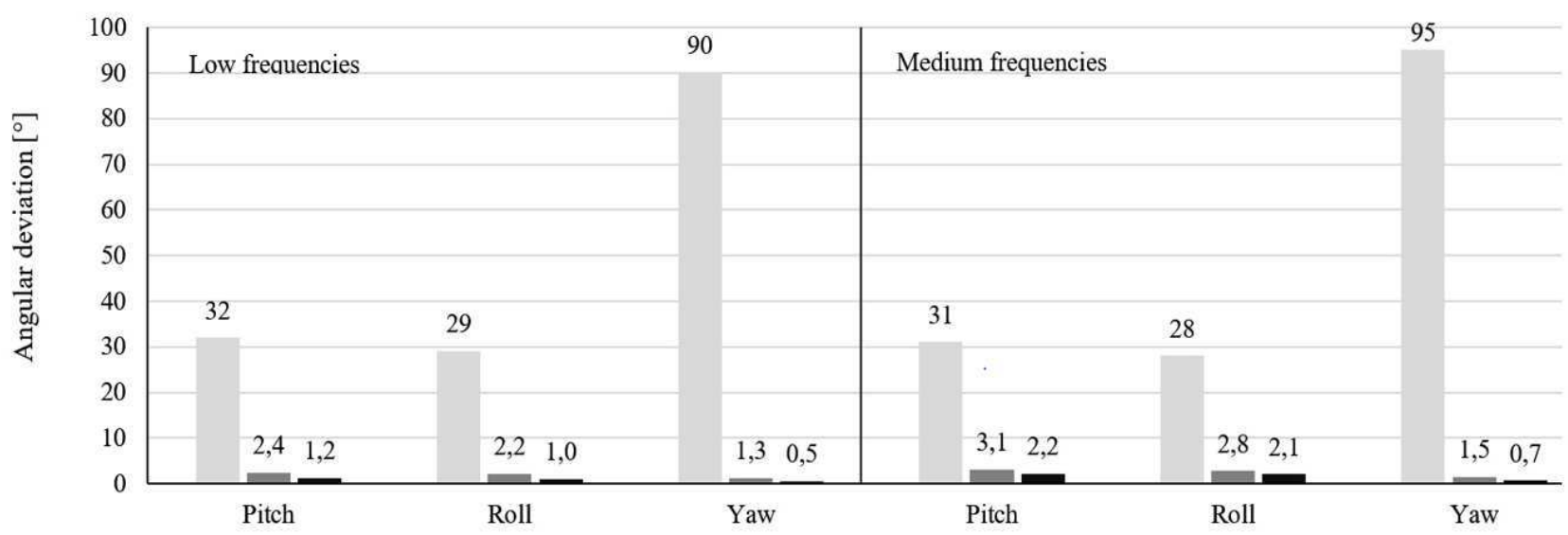

Figure 6: Angular deviation from gimbal in two frequencies 


\section{INSPECTION PROCESS WITH UAV}

The inspection process with UAV, which is shown below, is common to most applications in the field of civil engineering. Firstly, it is necessary to check and analyse the construction site, structure or area in order to locate the potential hazards. Special attention should be paid to safety requirements of the environment (people nearby, surrounding buildings, trees, weather conditions, especially wind and humidity, etc.) and the UAV status (GPS reception, condition of components, status of batteries, motors, $\mathrm{RC}$, etc.). Secondly, it is important to make a flight plan based on gathered information obtained at the site visit, to ensure that the pilot and the camera operator become familiar with predefined flight path, camera sequence and locations of image capturing, which are necessary for successful work completion. When those above-mentioned factors are in favour of safe flying, the inspection process should begin with the locating of the take-off point, which can be up to few hundred meters away from the inspecting location.

The take-off point should be on a flat surface to avoid and reduce chances of crash. Before flight, all vital components of the UAV must be checked in order to minimize chances of failures. After initialization of the UAV, the pilot can arm the aircraft and start to fly to the required inspection point and the camera operator may start capturing high-resolution images via remote control. With the help of the image and video transmitter, both, the pilot and the camera operator, are able to monitor the flight via live video transmission in order to see the desired details and monitor the location of flight. Furthermore, the operator is able to adjust the camera angle into the desired direction and to change basic parameters of the camera. The captured images are stored into an SD card and contain GPS coordinates, so the experts are able to relocate them for further use; for example, when a renewal of construction is necessary.

After the inspection process is finished, the pilot can fly the UAV back fully automatically to the starting point using smart features of the FCU. The UAV can also be operated via sticks on the $\mathrm{RC}$ and by the pilot himself. Post stabilization of pictures is not necessary, due to 3-axis camera stabilizing system IMU including dampers. Modern cameras also have integrated different mechanisms to stabilize the picture, so chances of blurred images are minimized. Transferred pictures can be analysed simultaneously by different experts directly from their offices. This feature represents one of the main advantages over the traditional inspection methods.

\section{PRACTICES AND FIELD APPLICATIONS OF THE UAV}

This section is devoted to present the applicability of the developed UAV. Due to the wide variety of possible uses, we decided to show some of the currently most frequent ones found in construction industry. Therefore, the continuation of this paper will show the uses of UAV that were actually performed in construction practice, i.e. construction site monitoring, examination of constructions, IR thermography, photogrammetry, transport applications and marketing activities.

\section{Construction site monitoring}

One of the field applications of the UAV is the construction site monitoring. The latter can represent a challenging task for a project manager for several reasons, such as complexity, risk of occupational injuries, frequency of visits, hard to reach locations and many more. Observing and monitoring the construction site from aerial viewpoint can bring positive effects on several segments; for example, reducing the time needed to patrol the site, the ability to see more details or to capture current conditions on the construction site for subsequent use.

The construction site monitoring begins with the flight of a multicopter equipped with a camera and a stabilizing system that can be controlled by an engineer. During the flight over a wide area it is possible to control the camera and with image transmission, live aerial picture can be seen on the screen by the pilot and the camera operator in real time. High-resolution images or videos are captured from the ground by using the radio remote control and are stored into the camera's hard drive for subsequent use. Described construction site monitoring certainly cannot replace the traditional workflows but can contribute to improve safety; for instance, to monitor the use of protective equipment, see Fig. 7. The main limitation of UAV-based construction site monitoring is in the project completion phase, where there are difficulties with the line of sight accessibility. 


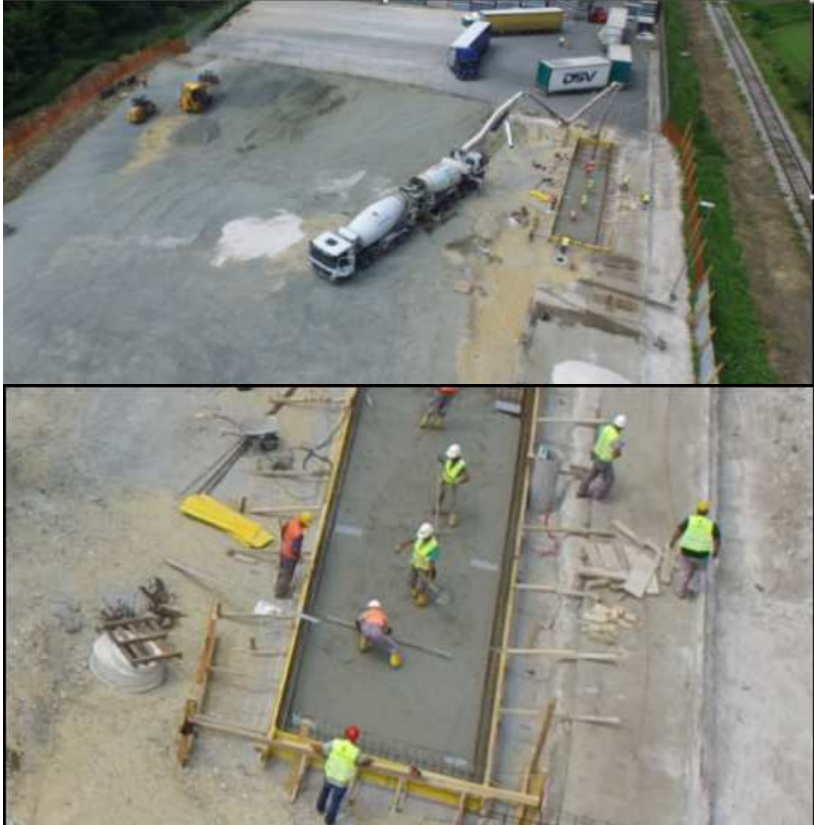

Figure 7: Aerial photograph of the construction site (upper); Magnified picture to monitor the use of protective equipment (lower)

\section{Examination of constructions}

Examination of different constructions in hard to reach locations often occurs in construction industry. Traditional methods for inspection of poorly accessible places may result in occupational injuries, increased costs due to the use of scaffoldings, ladders, lifts and other protective equipment and can cause the increased time required for work execution. Contemporary manner of constructions examination is possible with the help of modern UAVs from aerial viewpoint, without the need to bring scaffoldings and other protective equipment on work location, without organizing road closures and often even without a specialist for inspection on site, see Fig. 8.

The take off point of reviewing may start up to a few hundred meters away from the desired point of construction examination. In order to ensure greater safety and precision in managing the UAV during the flight it is recommended that in the reviewing process the pilot and the second operator of the camera cooperates to avoid possible faults. During the operation, both can employ live video transmission in order to see the details and monitor location of flight. Captured images or video clips may then be sent to different experts with the aim of simultaneous examination of the construction state. The presented way of examination reduces costs and time but cannot entirely replace existing methods due to several limitations. One of the main drawbacks is the fact that it is difficult to examine constructions above the aircraft due to the overlapped angle of the camera view.

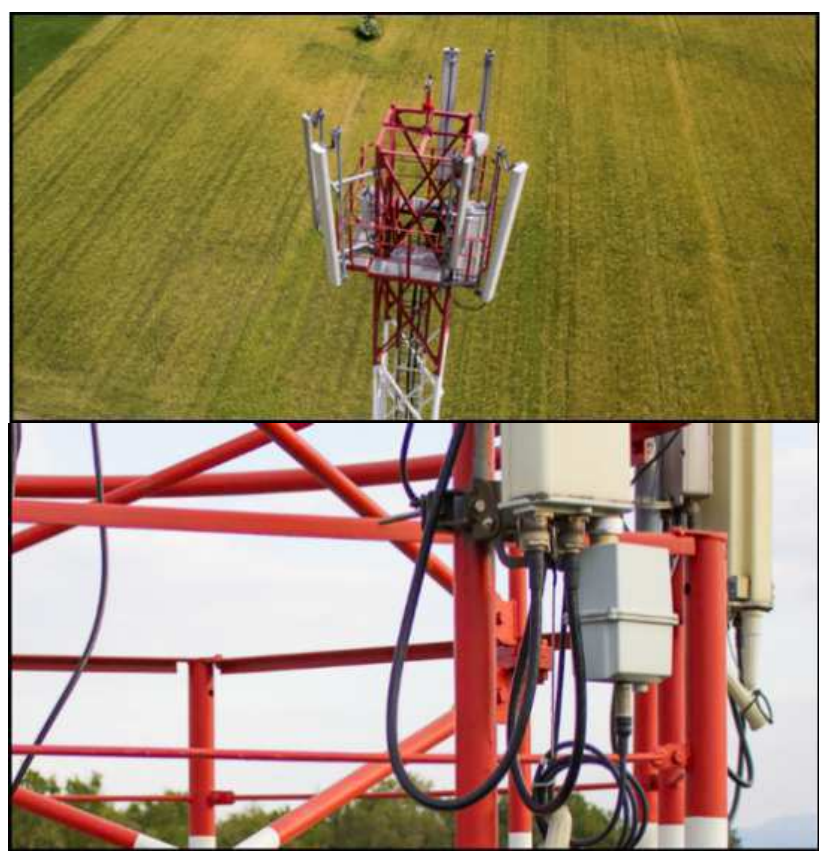

Figure 8: Examination of construction of the base station (upper); Detailed view of the construction

(lower)

\section{IR thermography}

Inspection of buildings and other objects with IR thermography may be helpful in different fields; two applications with UAV are presented below. An IR thermograph camera can be used in industry as beneficial tool for quality control [17]. That particularly comes to the fore in cases of special purpose buildings [18]. The IR cameras have become powerful, smaller and lightweight, so they can be used on UAVs. An IR thermocamera set on the UAV allows monitoring of the object temperature at a greater distance [19].

One of possible uses is scanning for faults on high-altitude electricity grids, which represents one of beneficial field usages of IR thermography performed with the UAV. At this point, locating defects on electrical network can be performed by scanning the area using the UAV equipped with the IR thermo-camera by which the camera operator is enabled to see and capture hot spots on the wires. Eventual deficiencies may be observed as brighter areas due to increased temperature compared to the surrounding areas. The main cause for higher temperature is often a damaged cable, where energy flow is raised due 
to smaller intersection of the cable what results in higher temperatures in the cable. The advantage, in addition to the faster and easier locating of faults, is also the possibility of inspecting electrical network without interruption of electricity supply.

Another field of the UAV application is identifying errors on photovoltaic panels, see Fig. 9. The production of electricity employing photovoltaics has become more popular and many owners as well as managers are curious about efficiency of their power plants. By using a UAV equipped with an IR thermo-camera, we are able to inspect large areas of photovoltaic power plants and to locate possible faults as well as to identify nonworking panels. The inspection process is similar to the one mentioned above. The operator is able to observe and locate possible errors on panels via live streaming picture from the air and also capture or record the area of plant. Eventual errors can usually be seen as bright spots and the temperature of a panel is not homogeneous. An important issue that should be taken into account in executing the aforesaid measurements in order to achieve precise recording is to select a suitable scanning angle, which should be between 70 and 90 degrees. Therefore, it is often difficult to gather such data adequately by using a handheld operated thermo-camera.

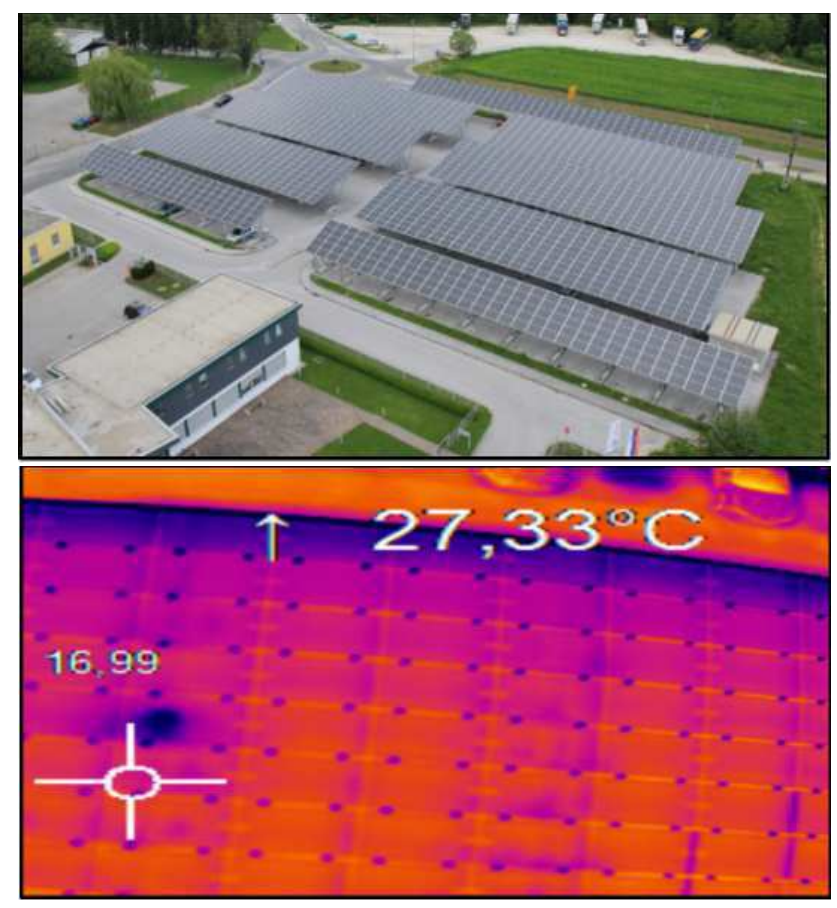

Figure 9: Common photo of photovoltaic power plant (upper); IR thermography review of photovoltaic power plant (lower)

\section{Photogrammetry}

In the construction branch, engineers are often faced with the calculation of surfaces and volumes. Many different calculation techniques exists in practice for such use. For example, one of the widely used approaches is calculating the volume by interpolating and summing up cross [20]. With the emergence of powerful software applications the photogrammetry became a useful tool for measuring areas or volumes on site, using a UAV equipped with a camera and a stabilizing system.

Photogrammetry is a technique where by pointing the camera vertically towards the ground, multiple overlapping photos of scanned terrain are taken during the flight of an aircraft and processed afterwards with suitable software, see Fig. 10. The software locates the camera position and orientation for each photo and thereupon stiches and aligns the overlapping photos into a model using a computer. After completion of this process, a sparse point cloud model is obtained.
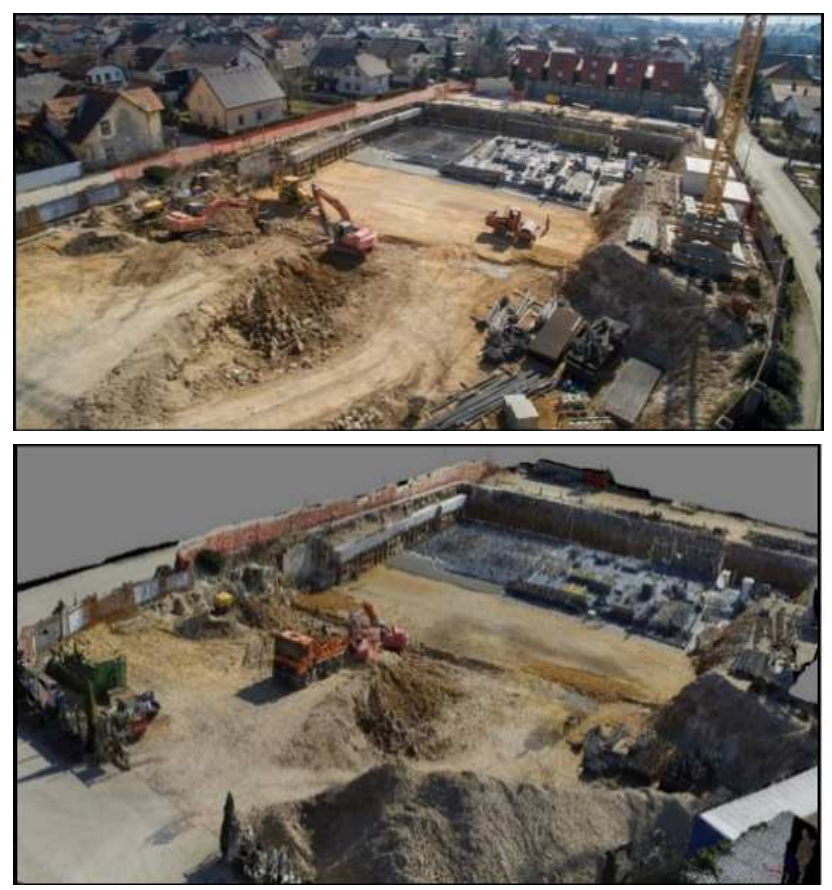

Figure 10: Realistic photography of construction site (upper); 3D model generated from aerial photos using the UAV (lower)

In the second step, building of a dense cloud is necessary, which is built on the calculated depth information for the camera in each position. The result is a single dense point cloud, where all gathered data is combined. Thereupon, in the third step, a mesh is created and after remov 
ing all secondary faces leaving only the surface to be considered in calculations, engineers are able to close holes of a model and measure volumes and areas, see Fig. 11. One of the main limitations is light condition, where pictures taking in low light situations can cause inaccurate measurements and errors in the model.
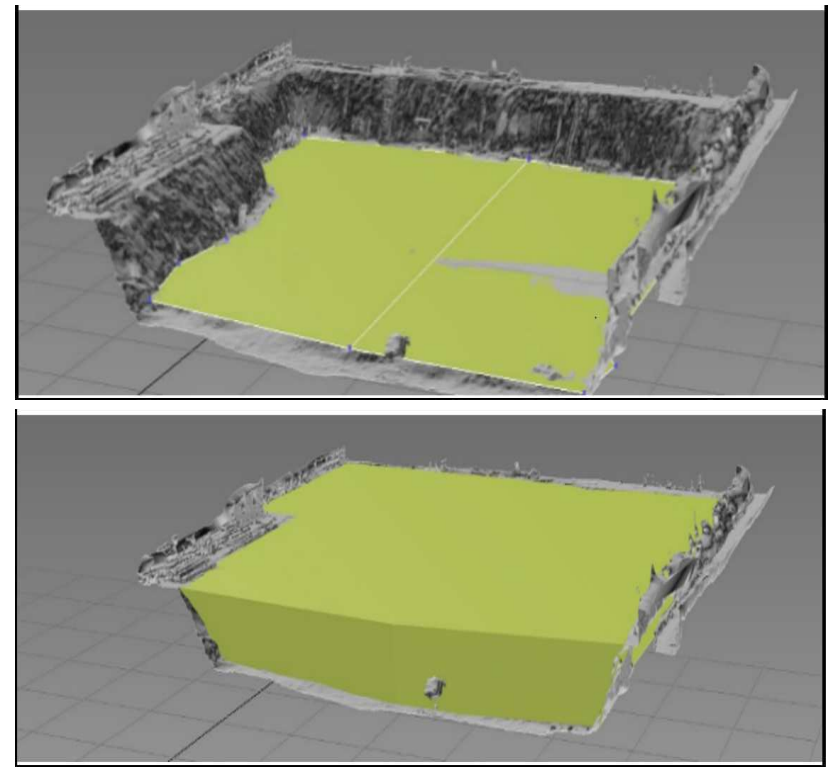

Figure 11: Area measurement of construction pit (upper); Volume measurement of excavated material (lower)

The photogrammetry can also be used in quarries, where there is a need for frequent measurements of stock, see Fig. 12. In the test case, we were measuring around $110.000 \mathrm{~m} 3$ of different aggregates in quarry. Stock was measured with traditional method as well, so a direct comparison between the results was obtained. Difference in measurement totalled $0.28 \%$, what represents minor difference and proves, that photogrammetry can be used in large scale measurements.

In addition, speed of measurement procedure is a key advantage of photogrammetry. Traditional methods require about 5 working days to complete this particular task, on the other side, only 2 working days were needed with the UAV. Furthermore the photogrammetry can also be used as an input to provide the data (as point cloud, DSM, orthomosaic) for spatial analysis; for example Geographic Information System (GIS). GIS is a powerful tool for spatial analysis and provides functionality to capture, store, query, analyse, display and output of geographic information [21].
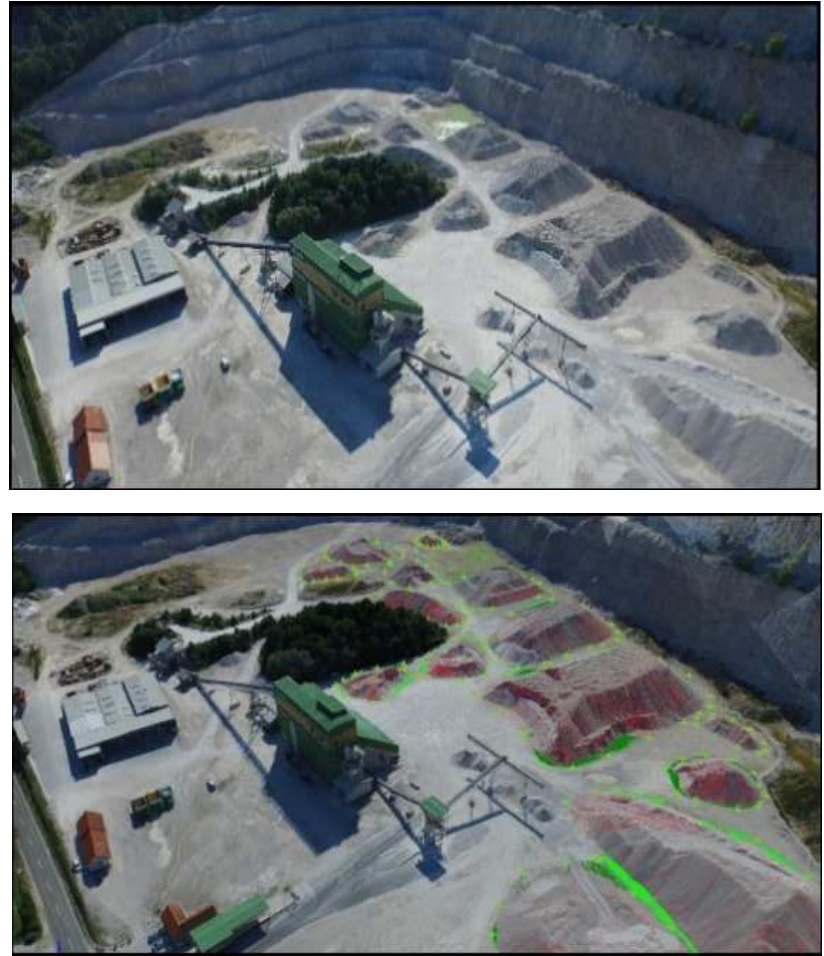

Figure 12: Realistic photography of quarry (upper); 3D model generated from aerial photos using the UAV for measuring stocks (lower)

\section{Transportation}

The development of new, powerful batteries and motors with greater efficiency enabled UAVs to become useful for transporting lighter loads to hardly accessible places. Recent multicopter systems can lift up to 20 kilograms or, in some cases even more with uprated electro motors, larger blades and reinforced frame. First examples of logistic applications are already taking place in Germany, where the transport company - DHL is shipping packages to island Juits at the edge of the Lower Saxon Wadden Sea in the southern North Sea [22].

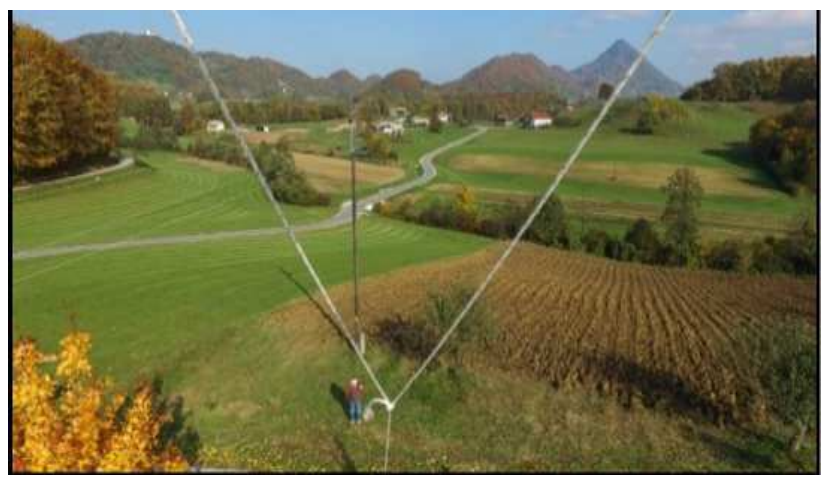

Figure 13. Transporting leader cable for electrical grid 
In cases of construction site transportations, it is clear that the UAVs cannot replace cranes or other important lifting devices but can be used as an auxiliary tool for the transferring lighter objects on locations that are hard to reach. For example, UAVs can be used for transporting the leader cable for electrical grids in hard to reach locations, for instance, high hills where use of conventional helicopters are often found to be quite expensive, see Fig. 13.

Here, UAVs may often prove to be capable enough to suitably transport required loads to such locations and, what should be highlighted - they manage to do it without environment contamination by exhaust gases or high noises during the flight.

\section{Marketing solutions}

UAVs can be also used for marketing purposes in various industries including the construction industry. Unmanned flying machines may be applicable for project presentations as well as introduction of reference objects. For example, Fig. 14 demonstrates a case of a photo presentation of successfully finished project - railway reconstruction [23].

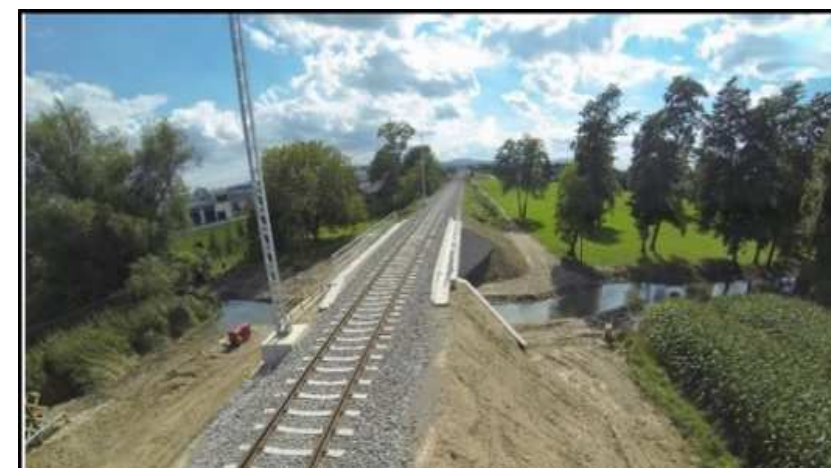

Figure 14. Showing a reference object for marketing purposes

Another possible marketing solution is sale promotion of real estate projects. By using UAVs, marketing experts are enabled to create wellprepared visual elements, such as images or videos for potential buyers. In most cases, money invested in marketing pays off, particularly for larger projects.

One of the innovative possibilities for marketing usage of the UAVs is promoting the company or a brand with attached advertising banner on ropes. This type of advertising can be prohibited in some countries; however, it normally attracts a lot of attention, especially in densely populated areas. On the other hand, this kind of marketing may constitute a potential danger to passers-by if not adequately organized and performed. For that purpose, it is generally advised that the UAV is equipped with more than four rotors to ensure greater safety and to reduce possibilities of failure.

\section{CONCLUSION}

This paper presented the UAV developed for multipurpose tasks in construction industry. The UAV was built as the hexacopter equipped with hardware and software systems that enable its multipurpose use at construction sites as well as on locations of existing facilities. The developed UAV was equipped with a specially designed stabilization system to achieve high quality photos or videos. Several actual field applications of the UAV were shown in order to demonstrate its advantages for construction practice.

Results obtained from the UAV during the flight showed differences in angular deviation between the bottom side of platform for electronics and gimbal. With two IMU gimbal controllers for alignments detection mounted and with the help of real time data from SimpleBGC software measuring was possible in all three axes and in two different frequency modes.

The effect of stabilization was visible in all aspects of many testing procedures including different applications from a photo camera to an IR thermo-camera. Furthermore, it was determined that the stabilization is crucial in ensuring high quality of capturing images or taking videos regardless to the used of camera technology. Namely, the impact of motor vibrations, wind and other factors can cause disturbances in image quality that result in blurred images.

Another important finding is that the UAV can be suitable for a multipurpose use, however it is essential to take the need for its adjustments into account. For instance, when applying different cameras it is necessary to recalibrate stabilization system for new weight and load distribution. The UAV can be helpful in construction industry on the account of cost savings, increased work efficiency, reduced occupational accidents frequency, and many more.

On the other hand, an important limitation for more frequent use in the field can be credited to relatively short flight time because of limited 
battery capabilities, engine's efficiency, weather dependence, and particularly, it is often a challenging task to implement new technologies into a traditional, proved well-functioning system of work activities in construction practice. Moreover, strict regulations already established in some countries may also represent additional barriers. Nevertheless, in the near future, we can expect a number of improvements of the basic components used in UAVs, especially batteries, motors and software, which will lead to new applications and will overcome today's limitations.

\section{REFERENCES}

1) Federal Aviation Administration: Small UAS Notice of Proposed Rulemaking (NPRM) https://www.faa.gov/uas/nprm/, retrieved on May 4th, 2016.

2) Schober, T. 2010. CLIBOT - a rope climbing robot for building surface inspection, Bautechnik, Vol. 87, No. 2, pp. 81-85.

3) Asbeck, A., Dastoor, S.; Parness, A.; et al. 2009. Climbing rough vertical surfaces with hierarchical directional adhesion, IEEE International Conference on Robotics and Automation, 12-17 May 2009, Kobe, pp. 2675-2680.

4) Haynes, G.C., Khripin, A., Lynch, G., et al. 2009. Rapid Pole Climbing with a Quadrupedal Robot, IEEE International Conference on Robotics and Automation, 12-17 May 2009, Kobe, pp. 2767-2772.

5) Metni, N., Hamel, T. 2007. A UAV for bridge inspection: Visual servoing control law with orientation limits, Automation in Construction, Vol. 17, No. 1, pp. 3-10.

6) Sa, I., Hrabar, S., Corke, P. 2014. Inspection of Pole-Like Structures Using a Vision-Controlled VTOL UAV and Shared Autonomy, IEEE International Conference on Intelligent Robots and Systems, 14-18 September 2014, Chicago, pp. 4819-4826.

7) Mejias, L., Correa, J., Mondragon, I., et al. 2007. COLIBRI: A vision-guided UAV for surveillance and visual inspection, IEEE International Conference on Robotics and Automation, 10-14 April 2007, Roma, pp. 2760-2761.

8) Roca, D., Lagueela, S., Diaz-Vilarino, L., et al. 2013. Low-cost aerial unit for outdoor inspection of building façades, Automation in Construction, Vol. 36, pp. 128-135.
9) Quater, P. B., Grimaccia, F., Leva, S., et al. 2014. Light Unmanned Aerial Vehicles (UAVs) for cooperative inspection of PV plants, IEEE Journal of Photovoltaics, Vol. , No. 4, pp. 1107-1113.

10)Moranduzzo, T., Melgani, F. 2014. Monitoring Structural Damages in Big Industrial Plants with UAV Images, IEEE International Symposium on Geoscience and Remote Sensing IGARSS, 13-18 July 2014, Quebec City, pp. 4950-4953.

11)Kharchenko, V., Prusov, D. 2012. Analysis of unmanned aircraft systems application in the civil field, Transport, Vol. 27, No. 3, pp. 335-343.

12)Cajzek, R., Klanšek, U. 2015. Applications of unmanned aerial vehicles in construction industry, 12th International Conference Organization, Technology and Management in Construction, 5-7 September 2015, Primošten, pp. 136-144.

13) Siebert, S., Teizer, J. 2014. Mobile 3D mapping for surveying earthwork projects using an Unmanned Aerial Vehicle (UAV) system, Automation in Construction, Vol.41, pp. 1-14.

14)Windau, J., Itti, L. 2011. Multilayer real-time video image stabilization, IEEE/RSJ International Conference on Intelligent Robots and Systems, 25-30 September 2011, San Francisco, pp. 2397-2402.

15)Åström, K. J., Hägglund, T. 2001. The future of PID control, Control Engineering Practice, Vol. 9, No. 11, pp. 1163-1175.

16)SimpleBGC. 2014. Software User Manual, Basecamelectronics. https://www.basecamelectronics.com/files/v10/SimpleBGC_manual_2_4_eng.pdf, retrieved on May 4th, 2016.

17) Vetter, A., Fecher, F., Adams, J., et al. 2013. Lock-in thermography as a tool for quality control of photovoltaic modules, Energy Science and Engineering, Vol. 1, No. 1, pp. 12-17.

18)Cajzek, R., Klanšek, U. 2014. Quality management of special purpose buildings: $A$ case of National Forensic Laboratory in Ljubljana, International Journal of Industrial Engineering and Management, Vol. 5, No. 3, pp. 115-122. 
19)Mavromatidis, L. E., Dauvergne, J. L., Saleri, R.; et al. 2014. First experiments for the diagnosis and thermophysical sampling using pulsed IR thermography from unmanned aerial vehicle (UAV), Quantitative InfraRed Thermography (QIRT) conference, 7-11 July 2014, Bordeaux, pp. 1-8.

20)Draeyer, B., Strecha, C. 2014. Putting stock in your survey, GEO: connexion, Vol.13, No. 2, pp. 32-34.

21)Rikalovic, A., Cosic, I., Lazarevic, D. 2014. GIS Based Multi-Criteria Analysis for Industrial Site Selection, Journal of Applied Engineering Science, Vol. 12, No. 3, pp. 197-206.
22)Heutger, M., Kückelhaus, M. 2014. Unmanned Aerial Vehicles in Logistics, DHL Customer Solutions \& Innovation. http://www. dhl.com/en/about_us/logistics_insights/dhl_ trend_research/uav.html, retrieved on May 4th, 2016.

23)Zajc, D., Bojc, I. 2014. Project documentation: Project Execution Plan, SŽ-projektivno podjetje Ljubljana d.d., Ljubljana 3639/OBJ_3/2

Paper sent to revision: 11.05.2016.

Paper ready for publication: 30.05.2016. 